\title{
Development and validation of a dietary screener for carbohydrate intake in endurance athletes
}

\author{
Stéphanie Harrison ${ }^{1,2}$, Élise Carbonneau ${ }^{1,2}$, Denis Talbot ${ }^{3}$, Simone Lemieux ${ }^{1,2}$ and Benoit Lamarche $e^{1,2^{*}}$ (D)
}

\begin{abstract}
Background: Studies have shown that the majority of endurance athletes do not achieve the minimal recommended carbohydrate $(\mathrm{CHO})$ intake of $6 \mathrm{~g} / \mathrm{kg}$ of body weight (BW), with potentially negative impacts on recovery and performance. The purpose of this study was to develop and validate a rapid and easy to use dietary screener to identify athletes who do and do not achieve a $\mathrm{CHO}$ intake $>6 \mathrm{~g} / \mathrm{kg}$ BW in the context of endurance sports.
\end{abstract}

Methods: The dietary screener was developed using multiple logistic regression modeling of data from a sample of 1571 non-athlete adults ( 826 women and 745 men, mean age $44.75 \pm 14.2$ years) among whom dietary intake was assessed using a validated web-based food frequency questionnaire (web-FFQ). Three models were developed based on whole food intake using the 5, 10 and 15 most significant variables predicting $\mathrm{CHO}$ intake. The three models were then validated in a target population of non-elite endurance athletes having taken part in multisport events ( $n=175$, 64 women and 111 men, mean age $37.1 \pm 11.3$ years) and compared using sensitivity, specificity, positive and negative predictive values (PPV and NPV, respectively) and c-statistics.

Results: The 15-variables model provided significantly better accuracy in predicting $\mathrm{CHO}$ intake adequacy in nonelite endurance athletes (c-statistic $=0.94)$ compared with the 10- and 5-variables model (c-statistic $=0.90$ and 0.71 respectively). The 15-variables model predicts $\mathrm{CHO}$ intake adequacy in the target population of endurance athlete with a sensitivity of $89.5 \%$, a specificity of $87.3 \%$ and PPV and NPV of 77.3 and $94.5 \%$, respectively.

Conclusion: We have successfully developed a short and valid dietary screener that identifies endurance athletes at risk of not achieving a $\mathrm{CHO}$ intake $>6 \mathrm{~g} / \mathrm{kg} \mathrm{BW}$. Use of this rapid screener may help alleviate the highly prevalent issue of suboptimal $\mathrm{CHO}$ consumption in the endurance sports realm.

Keywords: Carbohydrates, Dietary screener, Endurance athletes

\section{Background}

Carbohydrates (CHOs) are a crucial component of an athlete's diet, especially in endurance sports. Dietary CHOs contribute to restoring muscle and liver glycogen between training sessions and increase performance when their availability is maintained during the effort $[1,2]$. Recommendations on dietary $\mathrm{CHOs}$ are specific to sport, training regimen and competition schedule [2]. It is generally recommended that athletes consume between 6 and $10 \mathrm{~g}$ of

\footnotetext{
* Correspondence: benoit.lamarche@fsaa.ulaval.ca

${ }^{1}$ Institute of Nutrition and Functional Foods (INAF), Pavillon des Services, Laval University, 2440, Hochelaga Boulevard, Quebec City G1V 0A6, Canada

${ }^{2}$ School of Nutrition, Laval University, Quebec G1V 0A6, Canada

Full list of author information is available at the end of the article
}

CHO per kg of body weight (BW) per day when involved in an endurance program comprising moderate-to-high intensity trainings [1].

Multiple studies have shown that a large proportion of endurance athletes do not meet these recommendations. For instance, $45 \%$ of non-elite men and women participating in endurance multisport events such as IRONMAN triathlons were below the targeted $6 \mathrm{~g} \mathrm{CHO} / \mathrm{kg}$ of BW [3]. The prevalence of inadequate $\mathrm{CHO}$ intake was $80 \%$ among elite endurance athletes [4]. Studies have also shown that average $\mathrm{CHO}$ consumption among young pentathlon athletes and female collegiate athletes was below the recommendation $[5,6]$. This is an important

(C) The Author(s). 2018 Open Access This article is distributed under the terms of the Creative Commons Attribution 4.0 International License (http://creativecommons.org/licenses/by/4.0/), which permits unrestricted use, distribution, and 
concern because inadequate $\mathrm{CHO}$ consumption may lead to decreased work rates, impaired skills and concentration and increased effort perception, all of which are partly caused by fatigue [2]. Weakening of the immune system and increased risks of complications due to over-training have also been associated with low $\mathrm{CHO}$ intake in endurance athletes [7].

The difficulty in rapidly measuring food and nutrient intakes on the field certainly represents one of the most significant barriers to more optimal management of diet among athletes. Dietary assessment is costly and time-consuming and this is particularly troublesome in environments that generally rely on limited resources towards nutrition support. Having access to a rapid and cost-effective screening tool that identifies athletes at risk of not achieving dietary $\mathrm{CHO}$ recommendations will prove to be extremely useful to assign the limited nutrition support resources to those who need it the most. Therefore, the purposes of this study were 1) to develop a rapid and simple dietary screener predicting a $\mathrm{CHO}$ intake $>6 \mathrm{~g} / \mathrm{kg}$ of BW, which is considered the minimal recommendation for several endurance sports during moderate-intense training periods and 2) to validate and test the predictive value of the screener in a sample of endurance athletes. The screener was developed in a large sample of non-athletes to maximize statistical power and hence the stability of the predictive model. While we recognize that the $\mathrm{CHO}$ needs of a non-athlete population are very different than those of endurance athletes, we worked on the premise that the predictors of a high $\mathrm{CHO}$ intake are essentially the same in the two populations. We hypothesized that it is possible to identify endurance athletes at risk of not consuming adequate amounts of $\mathrm{CHO}$ based on a simple screening tool.

\section{Methods}

\section{Study participants}

A database of adult non-athlete subjects from previous projects conducted at the Institute of Nutrition and Functional Foods (INAF) in Quebec City was used to develop the screener (DEV sample). Multiple projects, in which subjects were all healthy, were included in the database. All participants provided consent in written form to have their data included in a database for use in research other than the main project to which they participated. The validity of the screener in the targeted population was assessed in a sample of non-elite endurance athletes (VALID sample). These athletes competed in Ironman triathlons (IM), Ironman 70.3 triathlons (IM 70.3 ), winter pentathlon (tandem or solo category) (9$15 \mathrm{~km}$ of cycling, $3.6-5.5 \mathrm{~km}$ of running, $4.9-8 \mathrm{~km}$ of cross-country skiing, $5-8.4 \mathrm{~km}$ of ice-skating, and 3.4$5.1 \mathrm{~km}$ of snowshoeing) or winter triathlon $(5 \mathrm{~km}$ of snowshoeing, $12 \mathrm{~km}$ of ice-skating, and $8 \mathrm{~km}$ of cross-country skiing). Non-elite athletes provided consent through an online system.

\section{Dietary data collection}

Participants in both the DEV and the VALID samples completed a validated web-based food frequency questionnaire (web-FFQ) [8]. This questionnaire contains 136 questions split into eight different sections: dairy products, fruits, vegetables, meat and alternatives, cereals and grain products, beverages, 'other foods' and dietary supplements. The web-FFQ inquires about food intake during the month prior to questionnaire completion. The Nutrition Data System for Research (software version 4.03, Food and Nutrient Database 31, Minneapolis, MN, USA) (Schakel et al., 1988) and the Canadian Nutrient File (CNF, version 2007b, Ottawa, ON, Canada) (Health Canada, 2007) were used to obtain nutrient intakes based on the answers provided in the web-FFQ. Food items from a broad category were grouped as one variable to simplify application and use of the final screener. For example, answers pertaining to brown and white rice consumption were added together to form only one category (frequency and amount of rice consumption).

\section{Model development}

Logistic regression modeling was used to develop the CHO-specific screener using data from the DEV sample. Analyses were undertaken in SAS (University Edition) unless stated otherwise. P-values less than 0.05 were considered statistically significant. Food servings per day, derived from the frequency and portion size of food categories in the web-FFQ, and participants' sex were considered in the primary phases of model development. Intake of individual macro- and micronutrients, such as lipids or proteins, was not considered because not all endurance athletes are aware of their specific nutrient consumption. Indeed, those at the higher end of the performance spectrum may be more meticulous in monitoring their dietary intake, hence having the knowledge and the resources to assess their own $\mathrm{CHO}$ intake. A screener for $\mathrm{CHO}$ intake is therefore not targeting these athletes, but rather those with limited resources and knowledge, who are less likely to monitor their diet and have a sense of their own $\mathrm{CHO}$ intake. Finally, dietary supplements were not considered, as data on sport-specific supplements were not available in the DEV sample.

Spearman's correlations between food intake (in servings/d) and $\mathrm{CHO}$ consumption (in $\mathrm{g} / \mathrm{kg}$ of $\mathrm{BW}$ ) were first calculated. The 25 foods showing the strongest univariate correlation with $\mathrm{CHO}$ consumption were retained. Then, for each of these 25 foods, cut-off points that best correlated with $\mathrm{CHO}$ intake above or below $6 \mathrm{~g} / \mathrm{kg} \mathrm{BW}$ were identified using logistic regression in R (version 3.3.0), in order to create dichotomic variables that can be answered simply by yes/no. These cut-off points were further adjusted to best reflect plausible daily or weekly servings. For example, the cut-off point 
for bread that best predicted a $\mathrm{CHO}$ intake $>6 \mathrm{~g} / \mathrm{kg}$ of BW in univariate logistic regression was 2.67 servings/d. This value was rounded up to 3 servings per day in order to facilitate the answering of the question by athletes. Next, multiple stepwise logistic regression models were constructed in SAS based on the 5, 10 and 15 variables that best predicted a $\mathrm{CHO}$ consumption $>6 \mathrm{~g} / \mathrm{kg}$ of BW. Contingency tables with derived sensitivity, specificity, positive and negative predictive values (PPV and $\mathrm{NPV}$, respectively) as well as receiver operating characteristic (ROC) curves and derived c-statistic were used to compare the performance of the three models. A model based on 20 variables or more was considered, but data indicated that model performance was no longer increased beyond 15 variables (not shown).

\section{Model validation}

Model validation was ascertained in the VALID sample of non-elite endurance athletes. The 5, 10 and 15-variables models derived from the development phase were compared for performance again using statistics from contingency tables as well as ROC curves.

\section{Results}

\section{Participants characteristics}

The DEV sample included 1571 participants (826 women and 745 men). Mean age was 44.8 years $(S D=14.3)$, mean body mass index (BMI) was $28.1 \mathrm{~kg} / \mathrm{m}^{2}(\mathrm{SD}=5.8)$ and mean $\mathrm{CHO}$ consumption was $3.75 \mathrm{~g} / \mathrm{kg}$ of $\mathrm{BW}(\mathrm{SD}=1.5)$. Only $7.2 \%$ of participants in this sample had a $\mathrm{CHO}$ consumption $>6 \mathrm{~g} / \mathrm{kg}$ of BW (Table 1 ).

The VALID cohort included 175 athletes (64 women and 111 men). Mean age was 37.1 years $(\mathrm{SD}=11.3)$, mean BMI was $23.3 \mathrm{~kg} / \mathrm{m}^{2}(\mathrm{SD}=2.6)$ and mean CHO consumption was $5.4 \mathrm{~g} / \mathrm{kg} \mathrm{BW}(\mathrm{SD}=2.5)$. A total of $32.6 \%$ consumed more than $6 \mathrm{~g}$ of $\mathrm{CHO} / \mathrm{kg}$ of $\mathrm{BW}$ (Table 2).

\section{Model development}

The 15-variables model showed the highest c-statistic $(0.89, p<0.004$ vs other models, Fig. 1$)$ with a sensitivity of $73.5 \%$, a specificity of $86.7 \%$, a NPV of $97.7 \%$ and a PPV of 30.0\% (Table 3).

Table 1 Characteristics of subjects in the DEV sample $(n=1571)$

\begin{tabular}{ll}
\hline Women, \% & $52.6 \%$ \\
Age, $y$ & $44.8 \pm 14.3^{\mathrm{a}}$ \\
Body weight, $\mathrm{kg}$ & $79.9 \pm 18.9$ \\
$\mathrm{BMI}, \mathrm{kg} / \mathrm{m}^{2}$ & $28.1 \pm 5.8$ \\
Carbohydrates consumption, g/kg of body weight & $3.75 \pm 1.5$ \\
Subjects consuming $>6 \mathrm{~g} \mathrm{CHO} / \mathrm{kg}$ of body weight, (\%) & $7.2 \%$ \\
\hline
\end{tabular}

${ }^{\mathrm{a}}$ Mean \pm SD (all such values) unless stated otherwise
Table 2 Characteristics of subjects in the VALID sample $(n=$ 175)

\begin{tabular}{|c|c|}
\hline Women, \% & $36.6 \%$ \\
\hline Age, $y$ & $37.1 \pm 11.3^{\mathrm{a}}$ \\
\hline Body weight, $\mathrm{kg}$ & $69.1 \pm 11.1$ \\
\hline $\mathrm{BMI}^{\mathrm{b}} \mathrm{kg} / \mathrm{m}^{2}$ & $23.3 \pm 2.6$ \\
\hline Carbohydrates consumption, $\mathrm{g} / \mathrm{kg}$ of body weight & $5.4 \pm 2.5$ \\
\hline Subjects consuming $>6 \mathrm{~g} \mathrm{CHO} / \mathrm{kg}$ of body weight, $\%$ & $32.6 \%$ \\
\hline
\end{tabular}

${ }^{\mathrm{a}}$ Mean $\pm \mathrm{SD}$ (all such values) unless stated otherwise

$\mathrm{b}_{n}=147$ because of 28 missing height values

\section{Model validation}

Table 4 shows the characteristics of the 5, 10 and 15 -variables models when applied to the target population of endurance athletes (VALID cohort), using the predetermined cut-offs for each food in the model. Consistent with data from the DEV sample, the 15-variables model performed significantly better than the 5 and 10 -variables models in predicting $\mathrm{CHO}$ intake (Fig. 2). The 15-variables model identified athletes achieving the minimal $\mathrm{CHO}$ recommendation (>6 g/ $\mathrm{kg}$ of BW) with a sensitivity of $89.5 \%$ and a specificity of $87.3 \%$. NPV and PPV were 94.5 and $77.3 \%$, respectively. Table 5 presents the final screener based on the 15-variables model, presenting each food retained in the model with its corresponding cut-off (formulated as a question) and their associated multivariate $\beta$ derived from the multivariate logistic model. These $B$ are used to define the predictive model that will be deployed to predict one's risk of not achieving the $\mathrm{CHO}$ recommendations for endurance sports.

\section{Discussion}

Rapid and cost-efficient assessment of $\mathrm{CHO}$ consumption among endurance athletes is challenging on the field. Although multiple dietary assessment tools, such as FFQs, $24 \mathrm{~h}$ recalls and dietary journals, are available to calculate an athlete's $\mathrm{CHO}$ intake, these tools usually take a lot of time to complete and require the experience of a trained professional for analysis. This, combined with the fact that large proportions of endurance athletes do not meet the recommended $\mathrm{CHO}$ intake, is a concerning issue. Here, we have developed a $\mathrm{CHO}$-specific dietary screener that allows rapid detection of endurance athletes at risk of not achieving a CHO intake of $6 \mathrm{~g} / \mathrm{kg}$ of BW or more. To our knowledge, this is the first validated tool that screens for adequate $\mathrm{CHO}$ intake among athletes.

The final model upon which the screener is based has both a high sensitivity and specificity in the target population (89.5 and $87.3 \%$, respectively), which are desired traits [9]. Such statistics indicate that the screener is as accurate in adequately identifying athletes who meet and those who do not meet the recommendation for $\mathrm{CHO}$ intake. The high AUC of the ROC curve (or c-statistic) 


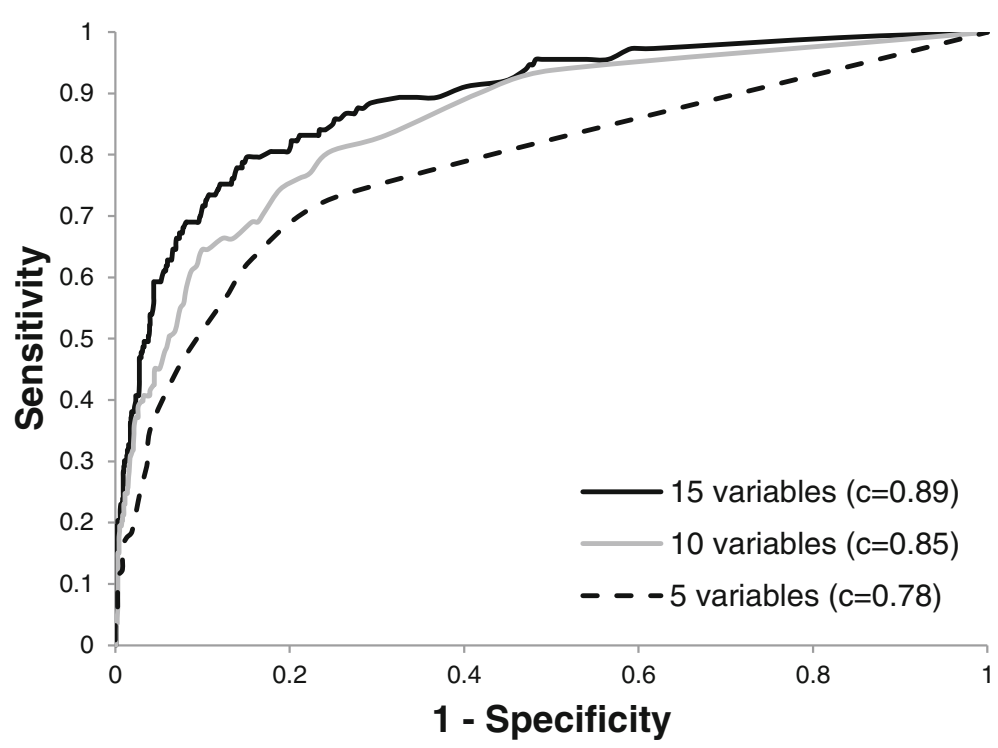

Fig. 1 ROC curves comparison of multiple logistic regression models in DEV sample. (c represents the c statistic on a scale of 0.5 to 1.0$)$

yielded by the 15 -variables model $(0.94$ on a range from 0.5 to 1.0$)$ is also reflective of a dietary screener that has excellent accuracy [9]. Furthermore, the model's NPV was considerably higher than its PPV (94.5\% vs. $77.3 \%)$, indicating that the screener is slightly more accurate in identifying endurance athletes who do not achieve adequate $\mathrm{CHO}$ intake than those who do. Such characteristic is highly desirable in the context of this research, as the ultimate goal of the $\mathrm{CHO}$ screener is to target athletes who would benefit from nutritional counseling, i.e. those with inadequate $\mathrm{CHO}$ intakes.

Very few studies have used an approach similar to ours to develop predictive models of adequate/inadequate dietary intakes, which makes comparison difficult. Most attempts were undertaken with a health rather than sports perspective. In those previous studies, predictive models and tools often achieved either a high sensitivity or a high specificity, but rarely both. For instance, Cook et al. built single-question and five-question screeners to rapidly assess fruits and vegetables intake among non-athletes. Sensitivity values ranged from 35.7 to $45.5 \%$ while specificity values ranged from of 81.8 to $84.9 \%$ among all five-question screeners developed by the research team. Using a single-question approach yielded high sensitivity but low specificity in the same population [10]. In most of these studies, the AUC of the ROC curves were fairly low, indicative of poor accuracy.

In an attempt to develop the simplest and yet most accurate $\mathrm{CHO}$ screener possible, we gave important considerations to limitations specific to the sports work environment. First, we had access to numerous dietary variables for the development of the model, such as energy, vitamin and protein intake, which may have contributed to a better prediction accuracy. However, such information is not readily available to either the respondent or the resource responsible for the screening test. It was therefore decided a priori to exclude such information. All anthropometric measures were also a priori excluded as they are too-closely related to the outcome measure to predict, which is based on BW. Similarly, cut-off values for each predictive food in the model were rounded to full daily or weekly servings to facilitate screener administration.

Several methods can be used to develop the predictive model of an outcome. Here, a multifaceted approach was used, but ultimately a stepwise logistic regression modeling approach yielded the final model. A classification tree $(\mathrm{CT})$ approach was also considered to develop the screener. This method uses discriminant analysis to

Table 3 Characteristics of the multiple logistic regression models in the DEV sample

\begin{tabular}{llllllll}
\hline Model & Sensitivity & Specificity & False positives & False negatives & PPV & NPV & c statistic \\
\hline 5 variables $^{\mathrm{a}}$ & 63.7 & 83.8 & 70.0 & 2.3 & 23.4 & 96.8 & 0.78 \\
10 variables & 64.6 & 87.7 & 71.0 & 3.0 & 29.0 & 97.0 & 0.85 \\
15 variables & 73.5 & 86.7 & 70.0 & 2.3 & 30.0 & 97.7 & 0.89 \\
\hline
\end{tabular}

$P P V$ positive predictive value, $N P V$ negative predictive value

a $\%$ (all such values) 
Table 4 Characteristics of the multiple logisitic regression models in VALID sampler

\begin{tabular}{llllllll}
\hline model & Sensitivity & Specificity & False positives & False negatives & PPV & NPV & c statistic \\
\hline 5 variables $^{\mathrm{a}}$ & 52.6 & 82.2 & 12.0 & 15.4 & 58.8 & 78.2 & 0.71 \\
10 variables & 75.4 & 86.4 & 9.1 & 8.0 & 72.9 & 87.9 & 0.90 \\
15 variables & 89.5 & 87.3 & 8.6 & 3.4 & 77.3 & 94.5 & 0.94 \\
\hline
\end{tabular}

PPV positive predictive value, NPV negative predictive value

a $\%$ (all such values)

test various combinations of variables in order to maximize the CT's predictive power [11]. Different algorithms can be used to build CTs; the CART algorithm was the chosen method for our purpose. What characterizes the CART algorithm is that it builds a very large $\mathrm{CT}$ and then prunes it to a smaller size to minimize classification errors [11]. A 10-fold cross-validation is used to prune the initial CT. The use of this method would have been beneficial for this particular research since ideal cut-off points are calculated directly in the CT algorithm. Unfortunately, this method yielded underwhelming results, with unacceptably high values for false negatives (approximately 30\%) when applied to the athletes sample (the VALID cohort). We hypothesize that the sample of non-athletes used to develop the CT may have comprised too few individuals with a $\mathrm{CHO}$ intake $>6 \mathrm{~g} / \mathrm{kg}$ of BW, thereby reducing the data usable by the algorithm to maximize the CT's predictive power.

Although this is the first study to develop a CHO-specific dietary screener for endurance athletes, limitations should be noted. First and foremost, the sample used to build the screener for application among athletes comprised non-athletes. This may have been a very significant shortcoming, considering that the diets of non-athletes and of endurance athletes are quite different. Second, a small proportion of the sample of individuals used to develop the screener achieved an intake of $\mathrm{CHO}$ greater than $6 \mathrm{~g} / \mathrm{kg}$ of BW, which may have hindered our ability to accurately predict this nutritional outcome. Ideally, the development of this CHO-specific screener would have been based on data from a large cohort of endurance athletes, but this was not possible. Third, the target of $6 \mathrm{~g} \mathrm{CHO} / \mathrm{kg} \mathrm{BW}$ may not be applicable to every endurance sport or training regimen, and this is a limitation when using the screener among athletes whose $\mathrm{CHO}$ needs are greater than $6 \mathrm{~g} / \mathrm{kg}$ of BW. Furthermore, participants in the development sample were not asked about $\mathrm{CHO}$ supplements often used in endurance sports. This is a limitation of the screener as intake of $\mathrm{CHO}$ is influenced by the use of such supplements in athletes. Nevertheless, the accuracy and hence validity of the $\mathrm{CHO}$-specific screener among endurance athletes is considered to be excellent, despite these limitations. Lastly, exploring different approaches for model development is a strength considering that very few studies in the field of nutrition have used CTs to create predictive models.

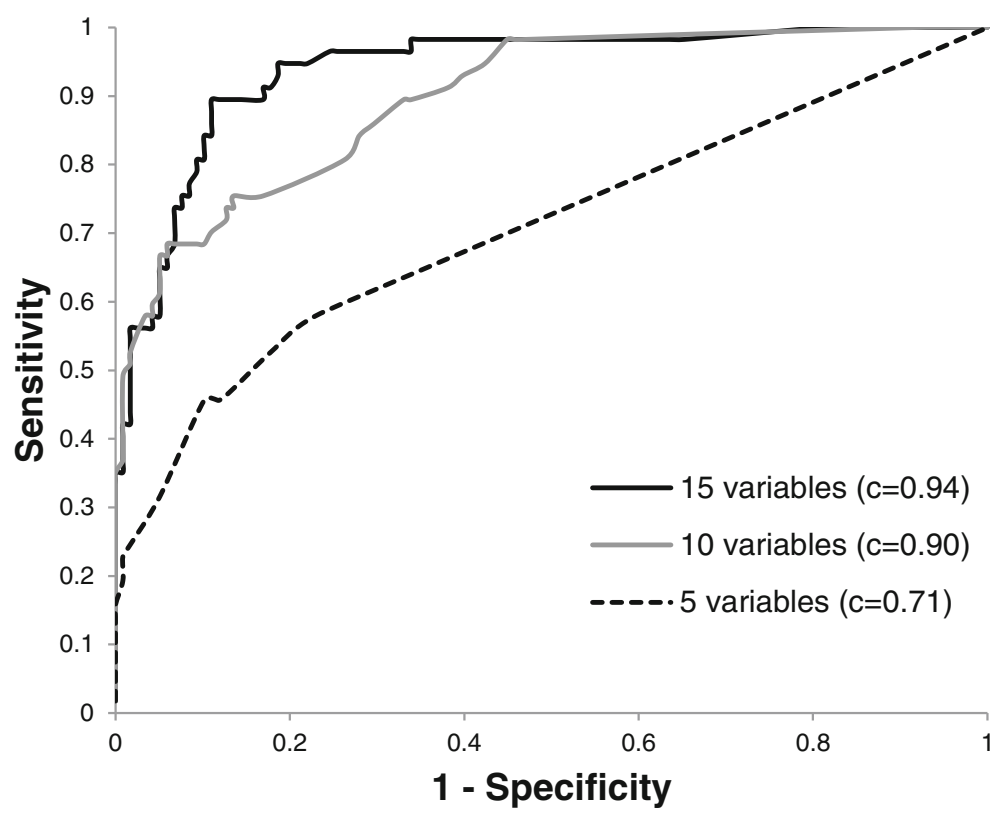

Fig. 2 ROC curves comparison of multiple logistic regression models in the VALID sample. (c represents the $c$ statistic on a scale of 0.5 to 1.0 ) 
Table 5 Final dietary screener

\begin{tabular}{|c|c|}
\hline Questions of the final dietary screener ${ }^{a}$ & ${ }^{b} \beta$ \\
\hline $\begin{array}{l}\text { Do you consume melons (watermelon, honeydew } \\
\text { r cantaloup) on a daily basis? }\end{array}$ & 0.5287 \\
\hline Do you consume pancakes twice a week? & 1.9666 \\
\hline o you consume avocado twice a week? & -0.0433 \\
\hline Do you consume cereal bars 6 times a week? & 2.0899 \\
\hline you consume rice 5 times a week? & 2.0401 \\
\hline o you drink chocolate milk 5 times a week? & 2.3249 \\
\hline o you consume chocolate (white, milk or dark) every week? & 1.8776 \\
\hline o you consume corn on a daily basis? & 0.7994 \\
\hline $\begin{array}{l}\text { o you consume milk, soy milk or silk tofu based } \\
\text { esserts } 3 \text { times a week? }\end{array}$ & 5.3373 \\
\hline Do you consume cold breakfast cereals on a daily basis? & 3.0771 \\
\hline Do you consume pasta on a daily basis? & 0.8276 \\
\hline $\begin{array}{l}\text { o you consume jam, maple by-products, hazelnut } \\
\text { oread, jelly or chocolate syrup twice a day? }\end{array}$ & 2.3477 \\
\hline Do you consume salad, lettuce or spinach twice a day? & 2.7638 \\
\hline drink soft drinks 3 times a day? & 10.3662 \\
\hline Are you a woman? & 0.3734 \\
\hline \multicolumn{2}{|c|}{$\begin{array}{l}\text { a Final questions are based on optimal cut-off points calculated by } \mathrm{R} \text { (version } \\
\text { 3.3.0) that were further adjusted to best fit a daily or weekly number of } \\
\text { servings. Cut-off points represent the number of servings of each specific food } \\
\text { that best predicted a CHO consumption }>6 \mathrm{~g} / \mathrm{kg} \text { of BW } \\
\mathrm{b}_{\beta} \text { from the multivariate logistic regression model for each dichotomic } \\
\text { variable (yes } / \mathrm{no} \text { ) in the final dietary screener. All } \beta \text { are significant }(P<0.05)\end{array}$} \\
\hline
\end{tabular}

\section{Conclusion}

In sum, we were successful in developing a simple, 15-questions dietary screening tool that predicts with accuracy an athlete's risk of achieving a dietary $\mathrm{CHO}$ target of $6 \mathrm{~g} / \mathrm{kg} \mathrm{BW}$ for endurance sports. Since the screener was validated in non-elite endurance athletes, further research should be conducted to test the accuracy of the screening tool among elite endurance athletes. The extent to which information on supplement use, particularly $\mathrm{CHO}$ supplements, improves prediction of total $\mathrm{CHO}$ intake by the screener needs to be investigated in future studies. Nevertheless, this easy-to-use screening tool will be a great asset to field work in sports nutrition as it rapidly identifies athletes who may benefit the most from receiving dietary counseling to optimize their diet.

\footnotetext{
Abbreviations

AUC: Area under the curve; BW: Body weight; $\mathrm{CHO}$ : Carbohydrate; CNF: Canadian nutrient file; CT: Classification tree; IM: IRONMAN triathlon; IM70.3: IRONMAN 70.3 triathlon; INAF: Institute of Nutrition and Functional Foods; NPV: Negative predictive value; PPV: Positive predictive value; ROC: Receiver operating characteristic; Web-FFQ: Web-based food-frequency questionnaire
}

\section{Funding}

The present study was supported in part by a grant from Canadian Institutes of Health Research (CIHR; grant no. FHG 129921) and by Laval University's Chair of Nutrition.

\section{Availability of data and materials}

Authors can confirm that all relevant data are included in the article and/or its supplementary information files.

\section{Authors' contributions}

$\mathrm{SH}$ did all of the statistical analyses and wrote the paper. All authors have read and approved the final manuscript.

\section{Ethics approval and consent to participate}

Laval University's Research Ethics Board has approved the secondary use of data from the various projects used in this study. All subjects from the different projects and included in these analyses also gave their consent for secondary use of their data.

\section{Consent for publication}

Not applicable.

\section{Competing interests}

The authors declare that they have no competing interests.

\section{Publisher's Note}

Springer Nature remains neutral with regard to jurisdictional claims in published maps and institutional affiliations.

\section{Author details}

${ }^{1}$ Institute of Nutrition and Functional Foods (INAF), Pavillon des Services, Laval University, 2440, Hochelaga Boulevard, Quebec City G1V 0A6, Canada.

${ }^{2}$ School of Nutrition, Laval University, Quebec G1V 0A6, Canada. ${ }^{3}$ Department of Social and Preventive Medicine, Laval University, Quebec G1V 0A6, Canada.

Received: 15 May 2018 Accepted: 7 September 2018

Published online: 14 September 2018

\section{References}

1. Burke LM, Hawley JA, Wong SH, et al. Carbohydrates for training and competition. J Sports Sci. 2011;29(Suppl 1):S17-27.

2. Thomas DT, Erdman KA, Burke LM. Position of the academy of nutrition and dietetics, dietitians of Canada, and the american college of sports medicine: nutrition and athletic performance. J Acad Nutr Diet. 2016;116:501-28.

3. Masson G, Lamarche B. Many non-elite multisport endurance athletes do not meet sports nutrition recommendations for carbohydrates. Appl Physiol Nutr Metab. 2016;41:728-34.

4. Baranauskas M, Stukas R, Tubelis $L$, et al. Nutritional habits among highperformance endurance athletes. Medicina (Kaunas). 2015;51:351-62.

5. Shriver LH, Betts NM, Wollenberg G. Dietary intakes and eating habits of college athletes: are female college athletes following the current sports nutrition standards? J Am Coll Heal. 2013;61:10-6.

6. Coutinho LA, Porto CP, Pierucci AP. Critical evaluation of food intake and energy balance in young modern pentathlon athletes: a cross-sectional study. J Int Soc Sports Nutr. 2016;13:15.

7. Gunzer W, Konrad M, Pail E. Exercise-induced immunodepression in endurance athletes and nutritional intervention with carbohydrate, protein and fat-what is possible, what is not? Nutrients. 2012;4:1187-212.

8. Labonte ME, Cyr A, Baril-Gravel L, et al. Validity and reproducibility of a webbased, self-administered food frequency questionnaire. Eur J Clin Nutr. 2012; 66:166-73.

9. Zou KH, O'Malley AJ, Mauri L. Receiver-operating characteristic analysis for evaluating diagnostic tests and predictive models. Circulation. 2007;115: $654-7$.

10. Cook A, Roberts K, O'Leary F, et al. Comparison of single questions and brief questionnaire with longer validated food frequency questionnaire to assess adequate fruit and vegetable intake. Nutrition. 2015;31:941-7.

11. Loh W-Y. Classification and regression trees. Wiley Interdisciplinary Reviews: Data Mining and Knowledge Discovery. 2011;1:14-23. 